\title{
Intelligent Traffic Light Controller Using IR Sensors for Vehicle Detection
}

\author{
Mr. Yogesh Shinde ${ }^{1}$, Miss. Hemlata Powar ${ }^{2}$ \\ Student Dept of E\&TC, DACOE Karad, Kolhapur University, India \\ Assistant Professor Dept of E\&TC, DACOE Karad, Kolhapur University, India
}

\begin{abstract}
The aim of project is to reduce the problem of traffic congestion which is becoming a very severe problem now a days. The present traffic light system consist of a per defined Hardware which has a fixed time for green light and red light .To optimize this problem made a framework for an intelligent traffic control system. Generally the conventional traffic light system is not depends upon the density of the traffic. So the purpose of a scheme in which the time period of green light and red light is assigned on the basis of the density of the traffic present at that time. This can be done by using IR sensors. Once the density is calculated, the glowing time of green light is assigned by the help of the microcontroller. The sensors which are present on either sides of the road detect the presence of the vehicles and sends the information to the microcontroller. On the basis of those information, micro-controller will decide the glowing time of green light and red light. It means that the timing of the traffic lights is set according to the density of the vehicles. This is going to be very helpful to minimize the traffic congestion and it has a scope in future.
\end{abstract}

Keywords: Density based traffic control, infra-red sensors, RF transmitter-receiver, microcontroller based algorithm, Vehicle stoppage beyond zebra crossing.

\section{INTRODUCTION}

Human is the creation of god. For ease and making life more comfortable, human being an introducing new technologies every day. This is why traffic congestion is increasing on the road day-by-day. As a result there are two main problems are arises No traffic, but still need to wait, Heavy traffic jams. These problems occur due to Fixed Control on Traffic. The Fixed Control on Traffic means not controlling the traffic according to the density, but in manner of programming which is already fixed in the system. To solve this problem of a fixed traffic light control system, introducing a traffic control system which is depend on the density for keeping control on the traffic. It is named as 'Intelligent traffic control system depend on density'. Intelligent traffic control system depend on density means, a system which can modulates itself according to the number of vehicles or can say density. Here IR sensors uses and IR sensor contains IR transmitter IR receiver in itself. These IR transmitter and IR

\section{LITERATURE REVIEW}

In the past the researchers had gone through different types of technologies. A brief survey of various solutions to the traffic congestion problems are presented. In this system AVR 32 micro-controller with programmable flash memory, built-in 8 channel analog to digital converter and IR sensors are used. The IR sensors are used to detect the presence of emergency vehicle and the microcontroller is designed to give a red signal to all the sides but one with the emergency vehicle.[1] In this system active RFID tag, wireless coordinator wireless router, GSM modems and monitoring station software are used. Here the wireless devices are mounted on either sides of the road and they collect the data from the active RFID tags. Through GSM, monitoring station will collect all the data and respond to the corresponding traffic signal.[2] In this a wireless sensor network is being used. To define the direction of any emergency vehicle, system uses a fuzzy and by collecting all the information central monitoring Display system gives the corresponding appropriate response.[3] Three main function of this project are as follows,

\section{Block Diagram}

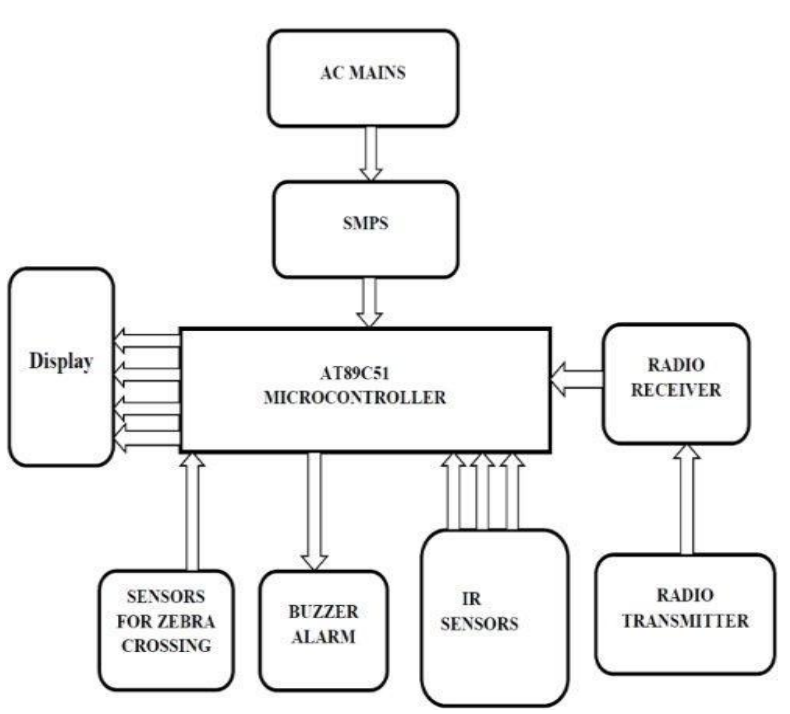

Fig 1 Functional Block Diagram 
Vol. 4, Special Issue 2, January 2017

\section{SYSTEM DESCRIPTION}

The basic block diagram of Intelligent Traffic Light Controller using IR sensors is shown in the above figure. Mainly this block diagram consists of the following essential blocks.

1. Density based traffic control

2. Immediately clearance to emergency vehicles

3. Detect vehicles on zebra crossing \& generate alarm

In this system three pairs of sensors across the roads marking as low, medium and high density zones respectively. There will be a infrared transmitter and infrared receiver opposite to each other. ir sensors place at some distance apart from another pair. When vehicles are filled and cross the first pair of sensors, then there will be an obstacle between transmitter and receiver and this make a digital signal (low or high) and the microcontroller assumes that there is low density traffic. When the vehicle crosses second sensor then it assumes medium density and for third sensor pair it assume as a high density traffic respectively.

Depending on the above process a digital data is sent to microcontroller whether it's low or high and the microcontroller will allot the time period for the traffic to pass on. For high density traffic allot more time period. allotment of time and for low density low time respectively. Program written to the microcontroller will make it to perform the operation. So the microcontroller will send its timing signal output by comparing with the adjacent road's traffic. The IR sensors also used to detect the presence of a vehicle over the zebra crossing when the signal is red. The microcontroller interface with an IR transmitter. The IR receiver was connected to a buzzer. If during red-light any vehicle tries to jump the red light or stops beyond the zebra crossing, then the buzzer will ring For the purpose of clearing way for the emergency vehicles, in this system use RF transmitter and receiver. An RF Transmitter will be placed on the special vehicles like fire brigade, ambulance, police or defence vehicles. The RF receivers will be placed before the signal that will receive the waves from the transmitter and detect the presence of the special vehicle and the signal will be transmitted to the microcontroller. In such a case, it will make the signal of that particular lane green and rest all red. The previous green lights in other lanes shall blink continuously indicating others of the special vehicle and fast clearing of vehicles before turning red.

AT89C51 series Microcontroller

RF Module

IR Sensors

Buzzer alarm

\section{MICROCONTROLLER AT89C51}

The AT89C51 is a low-power, high-performance CMOS 8-bit microcontroller with $4 \mathrm{~K}$ bytes of in-system programmable Flash memory. The device is manufactured using Atmel's high-density nonvolatile memory technology and is compatible with the industry-standard $80 \mathrm{C} 51$ instruction set and pin Out. The on-chip Flash allows the program memory to be reprogrammed insystem or by a conventional nonvolatile memory programmer.

By combining a versatile 8-bit CPU with in-system programmable Flash on a monolithic chip, the Atmel AT89S51 is a powerful microcontroller which provides a highly-flexible and cost-effective solution to many embedded control applications. The AT89S51 provides the following standard features: $4 \mathrm{~K}$ bytes of Flash, 128 bytes of RAM, $32 \mathrm{I} / \mathrm{O}$ lines, Watchdog timer, two data pointers, two 16-bit timer/counters, a five vector two-level interrupt architecture, a full duplex serial port, on-chip oscillator, and clock circuitry. In addition, the AT89S51 is designed with static logic for operation down to zero frequency and supports two software selectable power saving modes.

The Idle Mode stops the CPU while allowing the RAM, timer/counters, serial port, and

Interrupt system to continue functioning. The Power-down mode saves the RAM contents but freezes the oscillator, disabling all other chip functions until the next external interrupt or hardware reset.

\section{Features}

-Compatible with MCS-51® Products

-4K Bytes of In-System Programmable (ISP) Flash Memory Endurance: 1000 Write/Erase Cycles

$\bullet 4.0 \mathrm{~V}$ to $5.5 \mathrm{~V}$ Operating Range

-Fully Static Operation: $0 \mathrm{~Hz}$ to $33 \mathrm{MHz} \cdot$ Three-level

Program Memory Lock $\bullet 128$ x 8-bit Internal RAM

-32 Programmable I/O Lines

-Two 16-bit Timer/Counters $\bullet$ Six Interrupt Sources

-Full Duplex UART Serial Channel $\cdot$ Low-power Idle and

Power-down Modes $\bullet$ Fast Programming Time

-Flexible ISP Programming (Byte and Page Mode)

\section{IV.CONCLUSION}

This system conclude that using the method of density based control of traffic lights to save a considerable amount of time and also prevent the excessive traffic jams thus leading to smooth traffic flow.In India running system is time based to control the traffic signals and people experiencing a heavy traffic jams all over which in turn consumes lot of time and fuel. hope these methods will be earlier adopted so that the limitations are experiencing with present method can be overcome.

\section{ACKNOWLEDGMENT}

We would like to thank sincerely to our guide Miss. H.R.Powar and thank for endless support and motivation for her invaluable guidance, constant assistance, support, 
endurance and constructive suggestions for the betterment of this project work.

\section{REFERENCES}

[1] Sheela Joseph, Akhil S, Akhil K.Narayanankutty, Amalraj P.M. ,Aleena Paul K RF Transceiver Based Traffic Alert System for Automobiles, IJAREEIE, vol.2 issue 3, 2 Feb 2014.

[2] Intelligent Traffic Signal Control System by Dinesh Rotake \& Prof. Swapnil Karmore [1] (2012)

[3] This was presented in the Priority Based Traffic Lights Controller Using Wireless Sensor Networks by Shruthi K R and Vinod ha K [2](2012).

[4] Traffic congestion problem can also be reduced to greater extent through the process of image processing. This is mentioned in Image Processing Based Intelligent Traffic Controller by Dangi, Amol Parab, Vikram-aditya Kshitij Pawar and S. Rathod [4](2012).

[5] B. Prashanth Kumar, B. Karthik " Micro controller based traffic light controller", Department of Electrical \& Electronics Engineering Gokaraju Rangaraju Institute of Engineering \& Technology, 2011 ||

[6] Road Traffic Congestion Monitoring and Measurement using Active RFID and GSM Technology by Koushik Mandal, Arindam Sen, Abhijnan Chakraborty and Siuli Roy[3] (2011). 